\title{
Radiofrequency ablation in the management of Barrett's esophagus - preliminary own experience
}

\author{
Wojciech P. Dąbrowski, Andrzej B. Szczepanik, Andrzej Misiak, Konrad Pielaciński \\ Department of General and Hematological Surgery, Institute of Hematology and Transfusion Medicine, Warsaw, Poland
}

Videosurgery Miniinv 2013; 8 (2): 107-111 DOI: $10.5114 /$ wiitm.2011.32807

\begin{abstract}
Introduction: Barrett's esophagus develops as a result of chronic injury of esophagus epithelium from gastroesophageal reflux disease. It is defined when metaplastic columnar epithelium replaces the stratified squamous epithelium which normally lies in the distal esophagus. The condition represents a risk factor for esophageal adenocarcinoma. The aim of the radiofrequency ablation (RFA) method is to destroy metaplastic epithelium with radiofrequency electric current and to stimulate reappearance of the flat multilayer epithelium in the distal esophagus.

Aim: To evaluate the efficiency and safety of the RFA technique, newly introduced in Poland, in the management of Barrett's esophagus.

Material and methods: Twelve patients were treated with the RFA method. Patients with Barrett's esophagus confirmed in the histopathological report were qualified for treatment. Two RFA techniques were applied using a BARRX® device: circular based on the balloon HALO ${ }^{360}$ system or focal based on the HALO ${ }^{90}$ system mounted to the endoscopic ending. The procedures were performed at 2-month intervals. The macroscopic and microscopic effects of RFA therapy, the patients' treatment tolerance as well as potential complications were evaluated.

Results: In the group of 12 patients subjected to RFA therapy, 10 completed the therapeutic cycle. A total of 37 procedures were performed: $5 \mathrm{HALO} 360$ and $32 \mathrm{HALO}{ }^{90}$. In all patients eradication of the abnormal metaplastic esophageal epithelium was achieved, as confirmed in both endoscopic and histopathological evaluation. In 2 patients with ongoing therapy progressive eradication of metaplastic epithelium was observed. No significant RFA-related complications were reported.

Conclusions: Based on our preliminary results we consider this method to be promising, free of significant complications and well tolerated by patients. In most patients it results in successful eradication of metaplastic epithelium in the distal esophagus.
\end{abstract}

Key words: Barrett's esophagus, metaplasia, radiofrequency ablation.

\section{Introduction}

Barrett's esophagus (BE) develops as a result of chronic injury of esophagus epithelium from acid gastric content in the course of gastroesophageal reflux disease. Barrett's esophagus is defined when metaplastic columnar epithelium replaces the stratified squamous epithelium which normally lies in the distal esophagus. The condition represents a risk factor for esophageal adenocarcinoma. World statistics on the incidence of $\mathrm{BE}$ in the general population are divergent and vary between $0.9 \%$ and $6 \%[1]$. The annual incidence rate of adenocarcinoma in patients with $\mathrm{BE}$ is estimated at $0.5 \%$ [2]. The rise in incidence 
rate for esophageal adenocarcinoma is nowadays the highest among different types of carcinomas [3]. In the United States its incidence, estimated as $4 \%$ in the 1970 s, increased to $60 \%$ during the last thirty years [3]. Due to unsatisfactory Barrett's metaplasia recognition and the steadily growing number of newly diagnosed esophageal adenocarcinoma cases, this group of patients is steadily becoming the focus of attention.

During the last years several new methods for eradication of metaplastic epithelium in the distal esophagus have been developed. The most frequently applied methods are argon beam therapy, endoscopic mucosal resection, submucosal resection, cryotherapy, photodynamic therapy and, most recently introduced, radiofrequency ablation [4-7]. In this last method a radiofrequency electric current is applied to destroy metaplastic or dysplastic epithelium and to stimulate the process of flat multilayer epithelium reappearance [8].

\section{Aim}

The aim of the study was to evaluate the efficacy and safety of the radiofrequency ablation (RFA) method, newly introduced in Poland, for the management of $B E$.

\section{Material and methods}

Twelve patients (10 men, 2 women) aged 45-75 (average: 58.1 years) were treated with the RFA method in the period July 2010 - December 2011. Gastroesophageal reflux disease was diagnosed in all patients and sliding hiatus hernia in eight $(7$ men, 1 woman). Criteria for enrolment for Halo therapy were Barrett nondysplastic metaplasia, low grade dysplasia (LGD) or high-grade dysplasia (HGD) confirmed in histopathological reports. We also included 1 patient after endoscopic mucosal resection due to esophageal adenocarcinoma in situ. Radical excision with endoscopic mucosal resection was confirmed by histological evidence and endoscopic ultrasound investigation. Patients with esophagus mucosal injuries were disqualified but re-qualified after the lesions were cured.

Prior to therapy biopsy specimens were collected from all patients at $1 \mathrm{~cm}$ distance from four quadrants of the esophagus. Lesions were evaluated according to the Prague classification [9]. The proce- dures were applied in cycles at 2-month intervals under general anesthesia or intravenous premedication using the Halo device produced by BARRX Medical ${ }^{\circledR}$ (Sunnyvale, California, USA). Two RFA techniques were used depending on the type and location of metaplastic changes: circumferential or focal. The circumferential method based on a balloon system $\left(\mathrm{HALO}^{360}\right)$ was used for circular, metaplastic lesions on large surfaces, while the focal method based on the HALO ${ }^{90}$ system mounted on the endoscopic ending was used for smaller focal lesions. Radiofrequency energy was supplied to the tissue via a bipolar electrode; $12 \mathrm{~J} / \mathrm{cm}^{2}$ was applied for dysplastic lesions while $10 \mathrm{~J} / \mathrm{cm}^{2}$ was applied for intestinal metaplasia. The depth of ablative damage involved all the mucosa and the superficial part of submucosa. Not more than two $\mathrm{HALO}^{360}$ and three $\mathrm{HALO}^{90}$ procedures were performed per patient.

On the procedure day and during the next $24 \mathrm{~h}$ the patients were intravenously administered $40 \mathrm{mg}$ of omeprazole every $12 \mathrm{~h}$ and from the second day on they were given oral proton-pump inhibitors (PPI) doses of $40 \mathrm{mg}$ twice a day. On the procedure day the patients were administered fluids intravenously and a liquid diet was given for the next 2 days. After therapy all patients were subjected to 1-month endoscopic follow-up combined with histopathological examination. Macroscopic and microscopic effects of RFA were evaluated as well as patients' tolerance of the procedure and possible complications. Control examinations were supplemented by endoscopic evaluation with a narrow band imaging (NBI) system. Once the endoscopic treatment was completed patients were qualified for laparoscopic antireflux surgery. Four of them (4/11) expressed consent and were subjected to laparoscopic fundoplication with Nissen's method [10,11]. After the treatment course all patients were subjected to annual endoscopic and histopathological follow-up.

\section{Results}

In 10 of the 12 patients the treatment cycle was completed. The patients were subjected to a total of 37 procedures: 5 with $\mathrm{HALO}^{360}$ and 32 with $\mathrm{HALO}^{90}$. Prior to treatment BE nondysplastic metaplasia was diagnosed in 5 patients, LGD in 5 and HGD in 2 patients. The abnormal metaplastic esophageal epithelium was eradicated in all cases as confirmed in both the endoscopic and histopathological report. In 2 pa- 


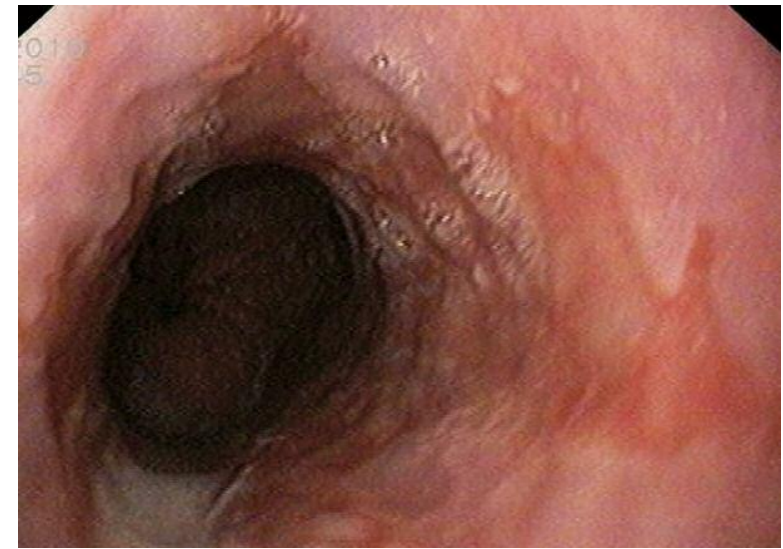

Photo 1. Barrett's esophagus; metaplastic epithelium focal areas visible in the lower part of the esophagus

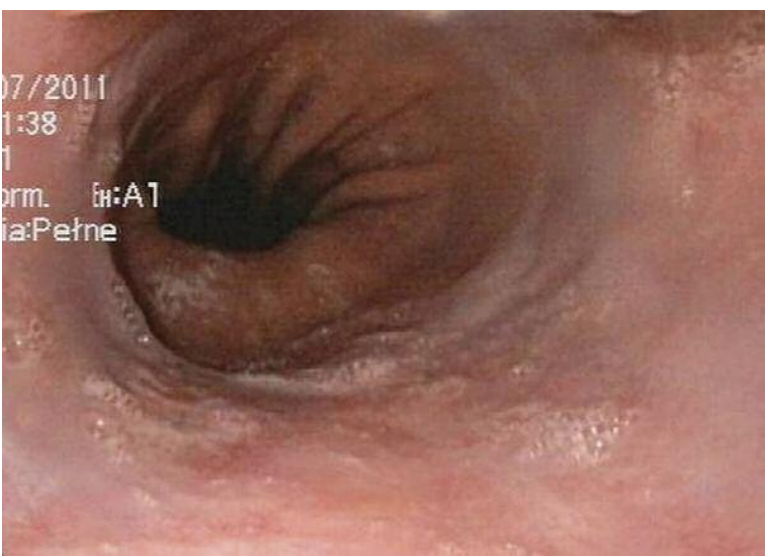

Photo 3. Endoscopic image after RFA treatment

tients with ongoing treatment the metaplastic epithelium was gradually eliminated. Two to five procedures (average: 3.5 ) were required to achieve squamous epithelium reappearance. Treatment duration ranged from 3 to 12 months (average: 6.4 months). No complications related to the RFA procedure were reported, which on the whole was well tolerated with only some epigastric and thoracic discomfort observed up to $12 \mathrm{~h}$ following the procedure. In 1 patient a minor focus of adenocarcinoma in the gastroesophageal junction 12 months after successful completion of treatment was discovered. The carcinoma texture was located below the normal epithelium. Endoscopic mucosal resection was performed. As a result of invasive cancer detected in histopathological examination the patient was qualified later for surgical resection.

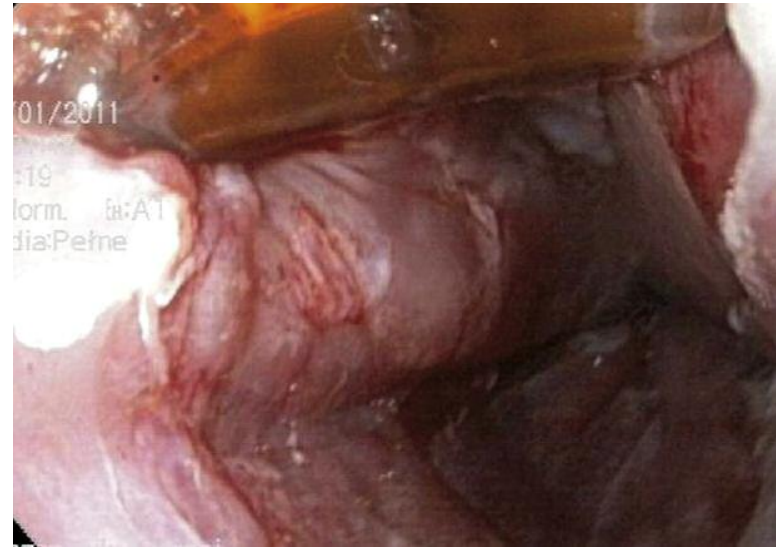

Photo 2. Therapy with BARRX ${ }^{\circledR} \mathrm{HALO}^{90}$ method

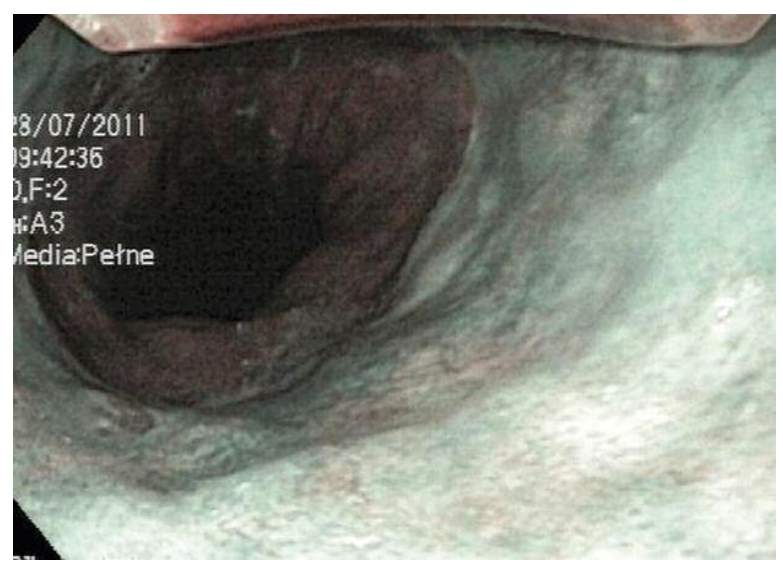

Photo 4. NBI endoscopic image after RFA treatment

\section{Discussion}

To date there are no univocal, consistent recommendations for the treatment of BE [12]. Despite the growing interest in the management of this condition in most countries the only recommendation for patients with BE is endoscopic surveillance with the aim of early recognition of cancer. The frequency of endoscopic follow-up depends on the type of lesions determined in histopathological examination. For isolated metaplasia endoscopy with biopsy is recommended every 2 years, for LGD every year and for HGD every 3-6 months [13]. Some authors also recommend resection procedures for patients with HGD. Numerous endoscopic and surgical methods of abnormal epithelium removal are currently being developed [12]. Antireflux surgery as prophylaxis against 


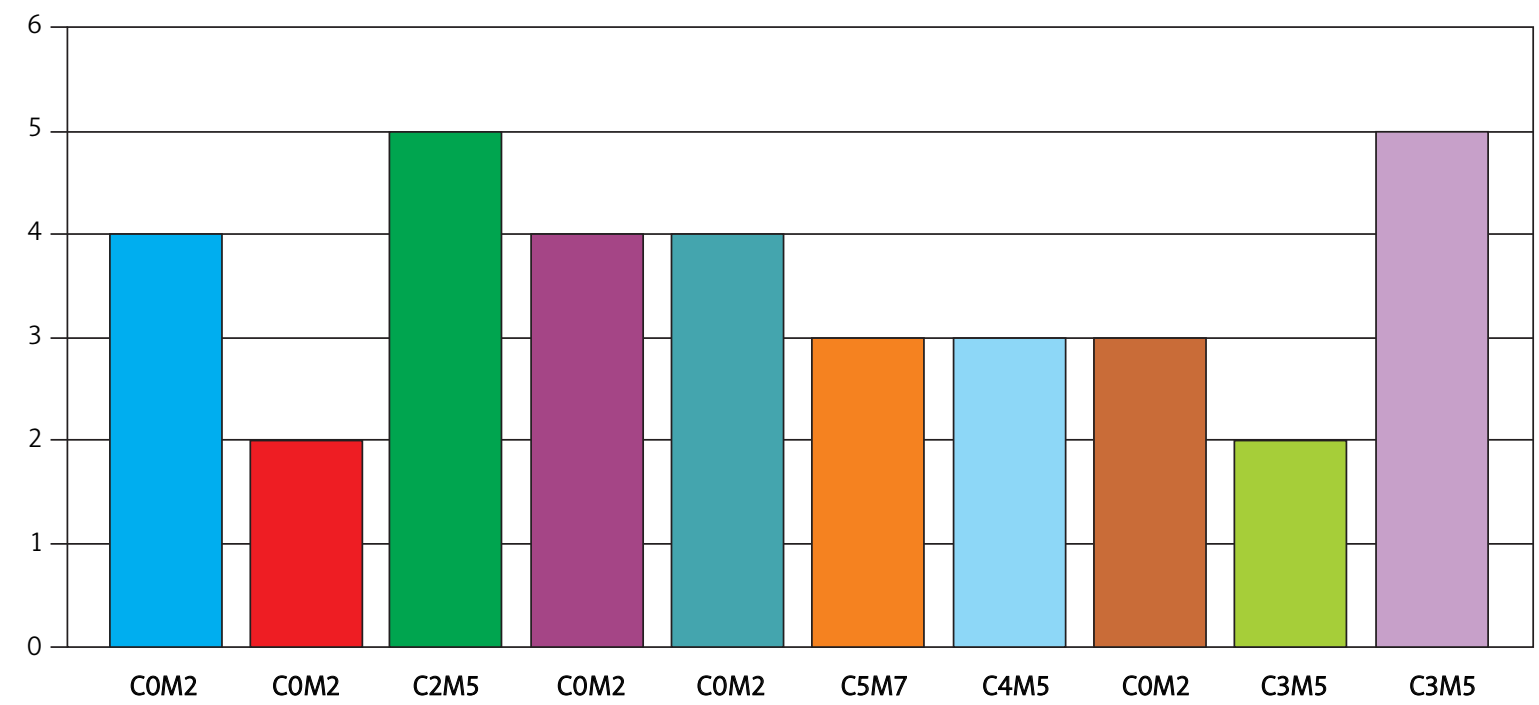

Prague classification: $C$ - circumference $(\mathrm{cm}), M$ - maximal extent $(\mathrm{cm})$

Figure 1. Number of HALO BARRX ${ }^{\circledR}$ procedures performed in consecutive patients according to Prague classification

reflux of gastric contents eliminates the underlying cause of BE $[12,14]$. In some patients however the metaplastic or dysplastic lesions persist and may lead to development of adenocarcinoma. According to some authors [14] these procedures should be combined with procedures applied for eradication of abnormal glandular epithelium such as argon coagulation or radiofrequency ablation. Following successful RFA 4 of our patients were subjected to antireflux surgery with good outcome.

Radiofrequency ablation is one of the most recent methods for management of BE. Data obtained from large clinical trials $[13,15]$ show this method to be $92 \%$ effective in the 5 -year follow-up of patients with nondysplastic metaplasia. For patients with lowgrade dysplasia it was proved effective in $81 \%$ of cases [13] and in $90 \%$ for patients with high-grade dysplasia $[16,17]$. The RFA method is almost as effective as endoscopic mucosal resection but the percentage of complications is lower [18]. The RFA-related complications include nausea, chest pain, transient dysphagia, esophageal wall injuries, stenosis, mediastinitis and esophageal perforation [19-21]. The frequency of RFA-related esophageal stenosis is estimated at $8 \%$ and is much lower than $88 \%$ after endoscopic mucosal resection [21].

No significant RFA-related complications were observed in our study group. This might be due to our restrictive qualification criteria which deferred patients with erosive injuries of esophageal mucosa. Performance of RFA in such patients might have led to esophageal perforation and higher risk of local stricture. It is equally important to avoid more than one ablation therapy within the same area during one procedure. Repeated ablation presents a similar complication risk as procedure performed in patients with mucosal injuries. Reliable information on the safety of this method can be possible only after analysis of a larger group of patients during a longer observation period. The reported case of a minor carcinoma focus in the gastro-esophageal junction located under macroscopically normal mucosa proves the need for regular and careful endoscopic surveillance and follow-up of this group of patients. It is worth noting that previous antireflux surgery and other surgical procedures in the area of the esophagus and mediastinum may impede RFA administration and in some cases (surgical clips and stapler stitches) may even inhibit this form of therapy. When planning to combine these therapeutic methods it is therefore mandatory to perform RFA as the first step and the antireflux procedure as the subsequent one.

\section{Conclusions}

Based on our preliminary results we consider this method to be promising, free of significant complica- 
tions and well tolerated by patients. It was successful for eradication of metaplastic epithelium in all of our patients. Endoscopic imaging after therapy showed no differences in physiological image. We cannot as yet evaluate this method in terms of prophylaxis against adenocarcinoma as this requires a long-term evaluation of a larger group of patients. All patients subjected to RFA procedures require further endoscopic surveillance and follow-up. The method can be combined with antireflux surgery. However, due to technical limitations the surgical procedures should be performed after completion of HALO therapy.

\section{References}

1. Ronkainen J, Aro P, Storksrubb T, et al. Prevalence of Barrett's esophagus in the general population: an endoscopic study. Gastroenterology 2005; 129: 1825-31.

2. Cameron AJ. Epidemiology of columnar-lined esophagus and adenocarcinoma. Gastroenterol Clin N Am 1997; 26: 487-94.

3. Pera M, Cameron AJ, Trastek VF, et al. Increasing incidence of adenocarcinoma of the esophagus and esophagogastric junction. Gastroenterology 1993; 104: 510-3.

4. Dąbrowski WP, Szczepanik AB, Misiak A. Radiofrequency ablation in the management of Barrett's esophagus - preliminary own experience. Pol Przegl Chir 2011; 3 (supl. 1): S44.

5. Konda VJ, Dalal K. Optimal management of Barrett's oesophagus: pharmacologic, endoscopic, and surgical interventions. Ther Clin Risk Manag 2011; 7: 447-58.

6. Sharma P, McQuaid K, Dent J, et al. A critical revive of the diagnosis and management of Barrett's esophagus. Gastroenterology 2004; 127: 310-30.

7. Van Vilsteren FG, Pouw RE, Seewald S, et al. Stepwise radical endoscopic resection versus radiofrequency ablation for Barrett's oesophagus with high-grade dysplasia or early cancer: a multicentre randomised trial. Gut 2011; 60: 765-73.

8. Bulsiewicz WJ, Shaheen NJ. The role of radiofrequency ablation in the management of Barrett's esophagus. Gastrointest Endosc Clin N Am 2011; 21: 95-109.

9. Anand O, Wani S, Sharma P. When and how to grade Barrett's columnar metaplasia: the Prague system. Best Pract Res Clin Gastoenterol 2008; 22: 661-9.

10. Vrba R, Aujesky R, Vomackova K, et al. Upside-down stomach results of mini-invasive surgical therapy. Videosurgery Miniinv 2011; 6: 231-5.

11. Szyca R, Leksowski K. Cornelia de Lange syndrome - characteristics and laparoscopic treatment modalities of reflux based on own material. Videosurgery Miniinv 2011; 6: 173-7.

12. Rembiasz K, Migaczewski M, Budzyński A, et al. Expression of cyclooxygenase- 2 in the mucosa of the gastroesophageal junction in patients with Barrett's oesophagus - the results of ablation therapy with argon plasma coagulation and laparoscopic Nissen fundoplication. Videosurgery Miniinv 2010; 5: 45-52.

13. Fleischer DE, Overholt BF, Sharma VK, et al. Endoscopic radiofrequency ablation for Barrett's esophagus: 5-year outcomes from a prospective multicenter trial. Endoscopy 2010; 42: 781-9.
14. Tarnowski W, Kiciak A, Borycka-Kiciak K, et al. Laparoscopic fundoplication improves oesophageal motility - a prospective study. Videosurgery Miniinv 2011; 6: 73-83.

15. Lyday WD, Corbett FS, Kuperman DA, et al. Radiofrequency ablation of Barrett's esophagus: outcomes of 429 patients from a multicenter community practice registry. Endoscopy 2010; 42: 272-8.

16. Ganz RA, Overholt BF, Sharma VK, et al. Circumferential ablation of Barrett's esophagus that contains high-grade dysplasia: a US Multicenter Registry. Gastrointest Endosc 2008; 68: 35-40.

17. Sharma VK, Wang KK, Overholt BF, et al. Balloon-based, circumferential, endoscopic radiofrequency ablation of Barrett's esophagus: 1-year follow-up of 100 patients. Gastrointest Endosc 2007; 65: 185-95.

18. Shaheen NJ, Sharma P, Overholt BF, et al. Radiofrequency ablation in Barrett's esophagus with dysplasia. N Engl J Med 2009; 360: 2277-88.

19. Fleischer DE, Overholt BF, Sharma VK, et al. Endoscopic ablation of Barrett's esophagus: a multicenter study with 2.5-year followup. Gastrointest Endosc 2008; 68: 867-76.

20. Yoon SS, Rivera R, Antignano L, Kaul V. A case of mediastinitis after radiofrequency ablation for Barrett's esophagus. Gastrointest Endosc 2011; 74: 1407-8.

21. Chennat J, Konda VJ, Ross AS, et al. Complete Barrett's eradication endoscopic mucosal resection: an effective treatment modality for high-grade dysplasia and intramucosal carcinoma an American single-center experience. Am J Gastroenterol 2009; 104: 2684-92.

Received: 24.08.2012, revised: 8.09.2012, accepted: 24.09.2012. 